\title{
On the Equivalence of Stochastic Fixed Point Iterations for Generalized $\varphi$-Contractive-Like Operators
}

\author{
Hudson Akewe, ${ }^{1}$ Kanayo Stella Eke, ${ }^{1}$ and Victoria Olisama $\mathbb{D}^{2}$ \\ ${ }^{1}$ Department of Mathematics, Covenant University, Canaanland, KM 10, Idiroko Road, PMB 1023, Ota, Ogun State, Nigeria \\ ${ }^{2}$ Department of Mathematics, Adeniran Ogunsanya College of Education, Otto-Ijanikin, Lagos, Nigeria \\ Correspondence should be addressed to Victoria Olisama; vicolisama@yahoo.com
}

Received 4 February 2018; Accepted 2 April 2018; Published 20 May 2018

Academic Editor: Shamsul Qamar

Copyright (C) 2018 Hudson Akewe et al. This is an open access article distributed under the Creative Commons Attribution License, which permits unrestricted use, distribution, and reproduction in any medium, provided the original work is properly cited.

We present the equivalence of some stochastic fixed point iterative algorithms by proving the equivalence between the convergence of random implicit Jungck-Kirk-multistep, random implicit Jungck-Kirk-Noor, random implicit Jungck-Kirk-Ishikawa, and random implicit Jungck-Kirk-Mann iterative algorithms for generalized $\varphi$-contractive-like random operators defined on separable Banach spaces.

\section{Introduction}

Probabilistic functional analysis is an aspect of mathematics that deals with probabilistic models to solve uncertainties and ambiguities that exist in real-world problems. Random nonlinear analysis is a vital area of probabilistic functional analysis that deals with various classes of random operator equations and related problems and solutions. The development of various random methods has transformed the financial markets. Random fixed point theorems for random contraction mappings on separable complete metric spaces were first proved by Spacek [1] and Hans (see [2, 3]). Random fixed point theory has become the full-fledged research area and various ideas associated with the theory are applied to obtain the solutions to a class of stochastic integral equations (see $[4,5])$. Random fixed theorems are well known stochastic generalizations of classical fixed point theorems and are usually needed in the theory of random equations, random matrices, random differential equations, and different classes of random operators emanating in physical systems $[6,7]$. The origin and various generalizations of random fixed point theorem exist in the literature; for complete survey, see [3] and several related references therein.

The concept of employing various iterative schemes in approximating fixed points of contractive-like operators is very useful in fixed point theory and applications and other relevant fields like numerical analysis, operation research, and so forth (see [8-15]) This is due to the close relationship that exists between the problem of solving nonlinear equations and that of approximating fixed points of corresponding contractive-like operator.

However, while many researchers have proved useful results on the equivalence of the various iterations, that is, the convergence of any of the iterative methods to the unique fixed point of the contractive operator for single map $T$ is equivalent to the convergence of the other iterations (see [16-24]), it is observed that little result is known of the equivalence of implicit schemes for a pair of maps [25]. This work will address these areas.

\section{Preliminary}

Definition 1. Let $(\Omega, \Sigma, \mu)$ be a complete probability measure space and $Y$ a nonempty subset of a separable Banach space $E$. For two random mappings $S, T: \Omega \times Y \rightarrow E$ with $T(\omega, Y) \subseteq$ $S(\omega, Y)$ and $C$ being a nonempty closed convex subset of a separable Banach space $E$, there exists a real number $\delta \in[0,1)$ and a monotone increasing function $\varphi: \mathbb{R}^{+} \rightarrow \mathbb{R}^{+}$with $\varphi(0)=0$, and for all $x, y \in C$, one has

$$
\begin{aligned}
\|T(\omega, x)-T(\omega, y)\| \leq & \delta\|S(\omega, x)-S(\omega, y)\| \\
& +\varphi(\|S(\omega, x)-T(\omega, y)\|),
\end{aligned}
$$


and for $x, y \in C$ and $h \in(0,1)$, one has

$$
\begin{gathered}
\|T(\omega, x)-T(\omega, y)\| \leq h \max \{\|S(\omega, x)-S(\omega, y)\|, \\
\frac{\|S(\omega, x)-T(\omega, x)\|+\|S(\omega, y)-T(\omega, y)\|}{2}, \\
\left.\frac{\|S(\omega, x)-T(\omega, y)\|+\|S(\omega, y)-T(\omega, x)\|}{2}\right\},
\end{gathered}
$$$$
\|T(\omega, x)-T(\omega, y)\| \leq h \max \{\|S(\omega, x)-S(\omega, y)\|,
$$$$
\frac{\|S(\omega, x)-T(\omega, x)\|+\|S(\omega, y)-T(\omega, y)\|}{2},
$$$$
\|S(\omega, x)-T(\omega, y)\|,\|S(\omega, y)-T(\omega, x)\|\} .
$$

For $x, y \in C, L>0,0<\delta<1$,

$$
\begin{aligned}
\|T(\omega, x)-T(\omega, y)\| \leq & \delta\|S(\omega, x)-S(\omega, y)\| \\
& +L\|S(\omega, x)-T(\omega, x)\| .
\end{aligned}
$$

For $x, y \in C, 0 \leq \delta<1, \quad M \geq 0$ and since $\varphi: \mathbb{R}^{+} \rightarrow \mathbb{R}^{+}$is a monotone increasing sequence with $\varphi(0)=0$, then

$$
\begin{aligned}
& \|T(\omega, x)-T(\omega, y)\| \\
& \quad \leq \frac{\delta\|S(\omega, x)-S(\omega, y)\|+\varphi(\|S(\omega, x)-T(\omega, x)\|)}{1+M\|S(\omega, x)-T(\omega, x)\|} \\
& \|T(\omega, x)-T(\omega, y)\| \\
& \quad \leq \delta\|S(\omega, x)-S(\omega, y)\|+\varphi(\|S(\omega, x)-T(\omega, x)\|) .
\end{aligned}
$$

Remark 2. Observe that the contractive conditions imply one another but not conversely: (2) $\Rightarrow(3) \Rightarrow(4) \Rightarrow(5) \Rightarrow(6)$.

Definition 3. Let $(\Omega, \Sigma)$ be a measurable space and $C$ be a nonempty closed convex sunset of a separable Banach space $E$. A function $T: \Omega \rightarrow C$ is said to be measurable if $T^{-1}(\mathscr{B} \cap$ $C) \in \sum$ for each Borel set $\mathscr{B}$ of $E$. A function $T: \Omega \times C \rightarrow C$ is called a random operator if $T(\cdot, x): \Omega \rightarrow C$ is measurable for every $x \in C$. A measurable function $f: \Omega \rightarrow C$ is called a random fixed point for the operator $T: \Omega \times C \rightarrow C$ if $T(\omega, f(\omega))=f(\omega)$.

Definition 4. Let $(\Omega, \Sigma)$ be a measurable space and $C$ be a nonempty closed convex subset of a separable Banach space $E$. A measurable function $f: \Omega \rightarrow C$ is called a random coincidence for two random mappings $S, T: \Omega \times C \rightarrow C$ if $T(\omega, f(\omega))=S(\omega, f(\omega))$ for all $\omega \in \Omega$. The maps $S, T$ are said to be random weakly compatible if they commute at their random coincidence; that is, if $S(\omega, f(\omega))=T(\omega, f(\omega))$ for every $\omega \in \Omega$, then $S(T(\omega, f(\omega)))=T(S(\omega, f(\omega)))$ or $S(\omega, T(\omega, f(\omega)))=T(\omega, S(\omega, f(\omega)))$.

We define the random explicit Jungck-Kirk-multistep hybrid iterative algorithm as follows.
Let $(\Omega, \Sigma)$ be a measurable space and $C$ be a nonempty closed convex subset of a separable Banach space $E$. Let $S, T$ : $\Omega \times C \rightarrow E$ be two random mappings with $T(\omega, C) \subseteq S(\omega, C)$. Let $x_{0}, y_{0}, z_{0}, u_{0}: \Omega \rightarrow C$ be an arbitrary measurable mapping for $\omega \in \Omega$, for $n=1,2, \ldots$.

(i) The random explicit Jungck-Kirk-multistep hybrid iterative algorithm is a sequence $\left\{S\left(\omega, x_{n}(\omega)\right)\right\}_{n=0}^{\infty}$ defined iteratively by

$$
\begin{array}{rl}
S\left(\omega, x_{n+1}(\omega)\right)= & \alpha_{n, 0} S\left(\omega, x_{n}(\omega)\right) \\
& +\sum_{i=1}^{k_{1}} \alpha_{n, i} T^{i}\left(\omega, y_{n}^{1}(\omega)\right), \\
S\left(\omega, y_{n}^{j}(\omega)\right)= & \beta_{n, 0}^{j} S\left(\omega, x_{n}(\omega)\right) \quad \alpha_{n, i}=1 \\
& +\sum_{i=1}^{k_{j+1}} \beta_{n, i}^{j} T^{i}\left(\omega, y_{n}^{j+1}(\omega)\right), \\
S\left(\omega, y_{n}^{q-1}(\omega)\right)= & \beta_{n, 0}^{q-1} S\left(\omega, x_{n}(\omega)\right) \\
& +\sum_{i=1}^{k_{j+1}} \beta_{n, i}^{q-1} T^{i}\left(\omega, x_{n}(\omega)\right), \\
& \sum_{n, i}^{j}=1, j=1,2, \ldots, q-2, \\
k_{q} & j=1, q \geq 2,
\end{array}
$$

where $k_{1} \geq k_{2} \geq k_{3} \geq \cdots \geq k_{q}, \alpha_{n, i} \geq 0, \quad \alpha_{n, 0} \neq 0, \quad \beta_{n, i}^{j} \geq$ $0, \beta_{n, 0}^{j} \neq 0$, for each $j$ and $\alpha_{n, i}, \beta_{n, i}^{j}$ are measurable sequences in $[0,1]$ for each $j$ while $k_{1}, k_{j}$ are fixed integers (for each $j$ ).

(ii) The random explicit Jungck-Kirk-Noor hybrid iterative algorithm is a sequence $\left\{S\left(\omega, y_{n}(\omega)\right)\right\}_{n=0}^{\infty}$ defined iteratively by

$$
\begin{aligned}
& S\left(\omega, y_{n+1}(\omega)\right)=\alpha_{n, 0} S\left(\omega, y_{n}(\omega)\right) \\
& +\sum_{i=1}^{k_{1}} \alpha_{n, i} T^{i}\left(\omega, y_{n}^{1}(\omega)\right) \\
& \sum_{i=1}^{k_{1}} \alpha_{n, i}=1 \\
& S\left(\omega, y_{n}^{1}(\omega)\right)=\beta_{n, 0}^{1} S\left(\omega, y_{n}(\omega)\right) \\
& +\sum_{i=1}^{k_{2}} \beta_{n, i}^{1} T^{i}\left(\omega, y_{n}^{2}(\omega)\right), \\
& \sum_{i=1}^{k_{2}} \beta_{n, i}^{1}=1
\end{aligned}
$$




$$
\begin{aligned}
S\left(\omega, y_{n}^{2}(\omega)\right)= & \beta_{n, 0}^{2} S\left(\omega, y_{n}(\omega)\right) \\
& +\sum_{i=1}^{k_{3}} \beta_{n, i}^{2} T^{i}\left(\omega, y_{n}(\omega)\right),
\end{aligned}
$$$$
\sum_{i=1}^{k_{3}} \beta_{n, i}^{2}=1
$$

where $k_{1} \geq k_{2} \geq k_{3}, \quad \alpha_{n, i} \geq 0, \alpha_{n, 0} \neq 0, \beta_{n, i}^{1} \geq 0, \beta_{n, 0}^{1} \neq 0$, $\beta_{n, i}^{2} \geq 0, \beta_{n, 0}^{2} \neq 0$, and $\alpha_{n, i}, \beta_{n, i}^{1}, \beta_{n, i}^{2}$ are measurable sequences in $[0,1]$ and $k_{1}, k_{2}, k_{3}$ are fixed integers.

(iii) The random explicit Jungck-Kirk-Ishikawa hybrid iterative algorithm is a sequence $\left\{S\left(\omega, z_{n}(\omega)\right)\right\}_{n=0}^{\infty}$ defined iteratively by

$$
\begin{aligned}
& S\left(\omega, z_{n+1}(\omega)\right)=\alpha_{n, 0} S\left(\omega, z_{n}(\omega)\right) \\
& +\sum_{i=1}^{k_{1}} \alpha_{n, i} T^{i}\left(\omega, z_{n}^{1}(\omega)\right) \\
& \sum_{i=1}^{k_{1}} \alpha_{n, i}=1 \\
& S\left(\omega, z_{n}^{1}(\omega)\right)=\beta_{n, 0}^{1} S\left(\omega, z_{n}(\omega)\right) \\
& +\sum_{i=1}^{k_{2}} \beta_{n, i}^{1} T^{i}\left(\omega, z_{n}(\omega)\right) \\
& \sum_{i=1}^{k_{2}} \beta_{n, i}^{1}=1
\end{aligned}
$$

where $k_{1} \geq k_{2} \alpha_{n, i} \geq 0, \alpha_{n, 0} \neq 0, \quad \beta_{n, i}^{1} \geq 0, \quad \beta_{n, 0}^{1} \neq 0$, and $\alpha_{n, i}, \beta_{n, i}^{1}$ are measurable sequences in $[0,1]$ and $k_{1}, k_{2}$ are fixed integers.

(iv) The random explicit Jungck-Kirk-Mann hybrid iterative algorithm is a sequence $\left\{S\left(\omega, u_{n}(\omega)\right)\right\}_{n=0}^{\infty}$ defined iteratively by

$$
\begin{aligned}
S\left(\omega, u_{n+1}(\omega)\right)= & \alpha_{n, 0} S\left(\omega, u_{n}(\omega)\right) \\
& +\sum_{i=1}^{k_{1}} \alpha_{n, i} T^{i}\left(\omega, u_{n}^{1}(\omega)\right),
\end{aligned}
$$

$$
\sum_{i=1}^{k_{1}} \alpha_{n, i}=1,
$$

where $\alpha_{n, i} \geq 0, \alpha_{n, 0} \neq 0$, and $\alpha_{n, i}$ is a measurable sequence in $[0,1]$ and $k_{1}$ is a fixed integer.

Next, we define the random implicit Jungck-Kirkmultistep hybrid iterative algorithms as follows.

Let $(\Omega, \Sigma)$ be a measurable space and $C$ be a nonempty closed convex subset of a separable Banach space $E$. Let $S, T$ : $\Omega \times C \rightarrow E$ be two random mappings with $T(\omega, C) \subseteq S(\omega, C)$. Let $x_{0}, y_{0}, z_{0}, u_{0}: \Omega \rightarrow C$ be an arbitrary measurable mapping for $\omega \in \Omega, n=0,1,2, \ldots$. (i) The random implicit Jungck-Kirk-multistep hybrid iterative algorithm is a sequence $\left\{S\left(\omega, x_{n}(\omega)\right)\right\}_{n=0}^{\infty}$ defined iteratively by

$$
\begin{aligned}
S\left(\omega, x_{n+1}(\omega)\right)= & \alpha_{n, 0} S\left(\omega, x_{n}^{(1)}(\omega)\right) \\
& +\sum_{i=1}^{k_{1}} \alpha_{n, i} T^{i}\left(\omega, x_{n+1}(\omega)\right),
\end{aligned}
$$$$
\sum_{i=1}^{k_{1}} \alpha_{n, i}=1
$$

$$
\begin{aligned}
S\left(\omega, x_{n}^{(j)}(\omega)\right)= & \beta_{n, 0}^{j} S\left(\omega, x_{n}^{(j+1)}(\omega)\right) \\
& +\sum_{i=1}^{k_{j+1}} \beta_{n, i}^{j} T^{i}\left(\omega, x_{n}^{j}(\omega)\right), \\
& \sum_{i=1}^{k_{j+1}} \beta_{n, i}^{j}=1, j=1,2, \ldots, q-2,
\end{aligned}
$$

$$
\begin{aligned}
S\left(\omega, x_{n}^{(q-1)}(\omega)\right)= & \beta_{n, 0}^{q-1} S\left(\omega, x_{n}(\omega)\right) \\
& +\sum_{i=1}^{k_{q}} \beta_{n, i}^{q-1} T^{i}\left(\omega, x_{n}(\omega)\right), \\
\sum_{i=1}^{k_{q}} \beta_{n, i}^{q-1} & =1, q \geq 2,
\end{aligned}
$$

where $k_{1}, k_{j}$ are fixed integers (for each $j$ ) with $k_{1} \geq k_{2} \geq$ $k_{3} \geq \cdots \geq k_{q}, \alpha_{n, i} \geq 0, \alpha_{n, 0} \neq 0, \beta_{n, i} \geq 0, \beta_{n, 0} \neq 0$, and $\alpha_{n, i}, \beta_{n, i}$ (for each $j$ ) are measurable sequences in $[0,1]$; (11) is called random implicit Jungck-Kirk-multistep hybrid iterative algorithm.

(ii) The random implicit Jungck-Kirk-Noor hybrid iterative algorithm is a sequence $\left\{S\left(\omega, y_{n}(\omega)\right)\right\}_{n=0}^{\infty}$ defined iteratively by

$$
\begin{aligned}
S\left(\omega, y_{n+1}(\omega)\right)= & \alpha_{n, 0} S\left(\omega, y_{n}^{(1)}(\omega)\right) \\
& +\sum_{i=1}^{k_{1}} \alpha_{n, i} T^{i}\left(\omega, y_{n+1}(\omega)\right), \\
& \sum_{i=1}^{k_{1}} \alpha_{n, i}=1 \\
S\left(\omega, y_{n}^{(1)}(\omega)\right)= & \beta_{n, 0}^{1} S\left(\omega, y_{n}^{(2)}(\omega)\right) \\
& +\sum_{i=1}^{k_{2}} \beta_{n, i}^{1} T^{i}\left(\omega, y_{n}^{(1)}(\omega)\right), \\
& \sum_{i=1}^{k_{2}} \beta_{n, i}^{1}=1,
\end{aligned}
$$




$$
\begin{aligned}
S\left(\omega, y_{n}^{(2)}(\omega)\right)= & \beta_{n, 0}^{2} S\left(\omega, y_{n}(\omega)\right) \\
& +\sum_{i=1}^{k_{3}} \beta_{n, i}^{2} T^{i}\left(\omega, y_{n}^{(2)}(\omega)\right), \\
& \\
& \sum_{i=1}^{k_{3}} \beta_{n, i}^{2}=1,
\end{aligned}
$$

where $k_{1} \geq k_{2} \geq k_{3}, \alpha_{n, i} \geq 0, \alpha_{n, 0} \neq 0, \beta_{n, i}^{1} \geq 0, \beta_{n, 0}^{1} \neq 0$, $\beta_{n, i}^{2} \geq 0, \beta_{n, 0}^{2} \neq 0$, and $\alpha_{n, i}, \beta_{n, i}^{1}, \beta_{n, i}^{2}$ are measurable sequences in $[0,1]$ and $k_{1}, k_{2}, k_{3}$ are fixed integers.

(iii) The random implicit Jungck-Kirk-Ishikawa hybrid iterative algorithm is a sequence $\left\{S\left(\omega, z_{n}(\omega)\right)\right\}_{n=0}^{\infty}$ defined iteratively by

$$
\begin{aligned}
& S\left(\omega, z_{n+1}(\omega)\right)=\alpha_{n, 0} S\left(\omega, z_{n}^{(1)}(\omega)\right) \\
& +\sum_{i=1}^{k_{1}} \alpha_{n, i} T^{i}\left(\omega, z_{n+1}(\omega)\right), \\
& \sum_{i=1}^{k_{1}} \alpha_{n, i}=1 \\
& S\left(\omega, z_{n}^{(1)}(\omega)\right)=\beta_{n, 0}^{1} S\left(\omega, z_{n}(\omega)\right) \\
& +\sum_{i=1}^{k_{2}} \beta_{n, i}^{1} T^{i}\left(\omega, z_{n}^{(1)}(\omega)\right), \\
& \sum_{i=1}^{k_{2}} \beta_{n, i}^{1}=1,
\end{aligned}
$$

where $k_{1} \geq k_{2}, \quad \alpha_{n, i} \geq 0, \quad \alpha_{n, 0} \neq 0, \quad \beta_{n, i}^{1} \geq 0, \quad \beta_{n, 0}^{1} \neq 0$, and $\alpha_{n, i}, \beta_{n, i}^{1}$ are measurable sequences in $[0,1]$ and $k_{1}, k_{2}$ are fixed integers.

(iv) The random implicit Jungck-Kirk-Mann hybrid iterative algorithm is a sequence $\left\{S\left(\omega, u_{n}(\omega)\right)\right\}_{n=0}^{\infty}$ defined iteratively by

$$
\begin{aligned}
S\left(\omega, u_{n+1}(\omega)\right)= & \alpha_{n, 0} S\left(\omega, u_{n}(\omega)\right) \\
& +\sum_{i=1}^{k_{1}} \alpha_{n, i} T^{i}\left(\omega, u_{n+1}(\omega)\right), \\
& \\
& \sum_{i=1}^{k_{1}} \alpha_{n, i}=1,
\end{aligned}
$$

where $\alpha_{n, i} \geq 0, \alpha_{n, 0} \neq 0$, and $\alpha_{n, i}$ are measurable sequences in $[0,1]$ and $k_{1}$ is a fixed integer.

Next, we introduce the random Jungck-Kirk-SP multiple iterative algorithm.

Let $(\Omega, \Sigma)$ be a measurable space and $C$ be a nonempty closed convex subset of a separable Banach space $E$. Let $S, T$ : $\Omega \times C \rightarrow E$ be two random mappings with $T(\omega, C) \subseteq S(\omega, C)$. Let $x_{0}, y_{0}: \Omega \rightarrow C$ be an arbitrary measurable mapping for $\omega \in \Omega, \quad n=0,1,2, \ldots$. (i) The random Jungck-Kirk-multistep-SP hybrid iterative algorithm is a sequence $\left\{S\left(\omega, x_{n}(\omega)\right)\right\}_{n=0}^{\infty}$ defined iteratively by

$$
\begin{aligned}
S\left(\omega, x_{n+1}(\omega)\right)= & \alpha_{n, 0} S\left(\omega, y_{n}^{(1)}(\omega)\right) \\
& +\sum_{i=1}^{k_{1}} \alpha_{n, i} T^{i}\left(\omega, y_{n}^{(1)}(\omega)\right),
\end{aligned}
$$

$$
\sum_{i=1}^{k_{1}} \alpha_{n, i}=1
$$

$$
\begin{aligned}
S\left(\omega, y_{n}^{(j)}(\omega)\right)= & \beta_{n, 0}^{j} S\left(\omega, y_{n}^{(j+1)}(\omega)\right) \\
+ & \sum_{i=1}^{k_{j+1}} \beta_{n, i}^{j} T^{i}\left(\omega, y_{n}^{(j+1)}(\omega)\right), \\
& \sum_{i=1}^{k_{j+1}} \beta_{n, i}^{j}=1, j=1,2, \ldots, q-2
\end{aligned}
$$

$$
\begin{aligned}
& S\left(\omega, y_{n}^{(q-1)}(\omega)\right)= \beta_{n, 0}^{q-1} S\left(\omega, x_{n}(\omega)\right) \\
&+\sum_{i=1}^{k_{q}} \beta_{n, i}^{(q-1)} T^{i}\left(\omega, x_{n}(\omega)\right), \\
& \sum_{i=1}^{k_{q}} \beta_{n, i}^{(q-1)}=1, q \geq 2,
\end{aligned}
$$

where $k_{1}, k_{j}$ are fixed integers (for each $j$ ) with $k_{1} \geq k_{2} \geq k_{3} \geq$ $\cdots \geq k_{q}, \alpha_{n, i} \geq 0, \alpha_{n, 0} \neq 0, \beta_{n, i} \geq 0, \beta_{n, 0} \neq 0$, and $\alpha_{n, i}, \beta_{n, i}$ (for each $j$ ) are measurable sequences in $[0,1]$.

(ii) The random Jungck-Kirk Noor-SP hybrid iterative algorithm is a sequence $\left\{S\left(\omega, y_{n}(\omega)\right)\right\}_{n=0}^{\infty}$ defined iteratively by

$$
\begin{aligned}
& S\left(\omega, y_{n+1}(\omega)\right)=\alpha_{n, 0} S\left(\omega, y_{n}^{(1)}(\omega)\right) \\
& +\sum_{i=1}^{k_{1}} \alpha_{n, i} T^{i}\left(\omega, y_{n}^{(1)}(\omega)\right) \\
& \sum_{i=1}^{k_{1}} \alpha_{n, i}=1 \\
& S\left(\omega, y_{n}^{(1)}(\omega)\right)=\beta_{n, 0}^{1} S\left(\omega, y_{n}^{(2)}(\omega)\right) \\
& +\sum_{i=1}^{k_{2}} \beta_{n, i}^{1} T^{i}\left(\omega, y_{n}^{(2)}(\omega)\right), \\
& \sum_{i=1}^{k_{2}} \beta_{n, i}^{1}=1
\end{aligned}
$$




$$
\begin{aligned}
S\left(\omega, y_{n}^{(2)}(\omega)\right)= & \beta_{n, 0}^{2} S\left(\omega, y_{n}(\omega)\right) \\
& +\sum_{i=1}^{k_{3}} \beta_{n, i}^{2} T^{i}\left(\omega, y_{n}(\omega)\right)
\end{aligned}
$$$$
\sum_{i=1}^{k_{3}} \beta_{n, i}^{2}=1,
$$

where $k_{1}, k_{2}, k_{3}$ are fixed integers with $k_{1} \geq k_{2} \geq k_{3}, \quad \alpha_{n, i} \geq$ $0, \alpha_{n, 0} \neq 0, \beta_{n, i} \geq 0, \beta_{n, 0} \neq 0$, and $\alpha_{n, i}, \beta_{n, i}$ (for each $j$ ) are measurable sequences in $[0,1]$.

Lemma 5. If $\delta$ is a real number such that $0 \leq \delta<1$ and $\left\{\varepsilon_{n}\right\}_{n=0}^{\infty}$ is a sequence of positive numbers such that $\lim _{n \rightarrow \infty}=0$, then, for any sequence of positive numbers $\{\varrho\}_{n=0}^{\infty}$ satisfying

$$
\varrho_{n+1} \leq \delta \varrho_{n}+\varepsilon_{n}, \quad n=0,1,2, \ldots,
$$

one has $\lim _{n \rightarrow \infty} \varrho_{n}=0$.

Lemma 6. Let $(X\|\cdot\|)$ be a normed linear space and $S, T: Y \rightarrow$ $X$ be a non-self-random commuting operator on an arbitrary set $Y$ with values in $X$ satisfying (6) such that $T(\omega, Y) \subseteq$ $S(\omega, Y)$ :

$$
\begin{aligned}
& \|S(\omega, S(\omega, x))-T(\omega, S(\omega, x))\| \\
& \quad \leq\|S(\omega, x)-T(\omega, x)\|, \\
& \|S(\omega, S(\omega, x))-S(\omega, S(\omega, y))\| \\
& \quad \leq\|S(\omega, x)-S(\omega, y)\| .
\end{aligned}
$$

Let $\varphi: \mathbb{R}^{+} \rightarrow \mathbb{R}^{+}$be a sublinear, monotone increasing function such that $\varphi(0)=0$ and $\varphi(u)=(1-\delta) u$ for all $0 \leq \delta<1, u \in$ $\mathbb{R}^{+}$. Then, for every $i \in \mathbb{N}$ and $x, y \in Y$, we have

$$
\begin{aligned}
\left\|T^{i}(\omega, x)-T^{i}(\omega, y)\right\| \\
\leq \delta^{i}\|S(\omega, x)-S(\omega, y)\| \\
\quad+\sum_{j=0}^{i}\left(\begin{array}{l}
i \\
j
\end{array}\right) \delta^{i-j} \varphi^{j}(\|S(\omega, x)-T(\omega, x)\|) .
\end{aligned}
$$

Proof. Lemma 6 is proved by mathematical induction in [17].

\section{Main Results}

Theorem 7. Let $E$ be a separable Banach space and $S, T$ : $\Omega \times E \rightarrow E$ be two random commuting mappings satisfying (19) such that $T(\omega, E) \subseteq S(\omega, E)$. Assume that $S$ and $T$ are random weakly compatible. Let $p(\omega)$ be the random common point of $S, T, S^{i}, T^{i}$ (i.e., $\left.S(\omega, p(\omega))=T(\omega, p(\omega))=p(\omega)\right)$. If $u_{0}, x_{0} \in$ $E$ defined by $\left\{S\left(\omega, u_{n}(\omega)\right)\right\}_{n=0}^{\infty},\left\{S\left(\omega, x_{n}(\omega)\right)\right\}_{n=0}^{\infty}$ as sequences satisfying (14) and (11), respectively, then the following are equivalent:

(i) Random implicit Jungck-Kirk-Mann iteration (14) converges strongly to $p(\omega)$.

(ii) Random implicit Jungck-Kirk-multistep iteration (11) converges strongly to $p(\omega)$.

Proof. We first prove that (i) $\Rightarrow$ (ii).

Assume $\lim _{n \rightarrow \infty} S\left(\omega, u_{n}(\omega)\right)=p(\omega)$, and then using (14) and (11) and generalized contractive condition (19), we get

$$
\begin{aligned}
\left\|S\left(\omega, u_{n+1}(\omega)\right)-S\left(\omega, x_{n+1}(\omega)\right)\right\| \leq & \alpha_{n, 0}\left\|S\left(\omega, u_{n}(\omega)\right)-S\left(\omega, x_{n}^{(1)}(\omega)\right)\right\|+\sum_{i=1}^{k_{1}} \alpha_{n, i}\left\|T^{i}\left(\omega, u_{n+1}(\omega)\right)-T^{i}\left(\omega, x_{n+1}(\omega)\right)\right\| \\
\leq & \alpha_{n, 0}\left\|S\left(\omega, u_{n}(\omega)\right)-S\left(\omega, x_{n}^{(1)}(\omega)\right)\right\| \\
& +\left(\sum_{i=1}^{k_{1}} \alpha_{n, i} \delta^{i}\right)\left\|S\left(\omega, u_{n+1}(\omega)\right)-S\left(\omega, x_{n+1}(\omega)\right)\right\| \\
& +\sum_{i=1}^{k_{1}} \alpha_{n, i}\left(\sum_{j=0}^{i}\left(\begin{array}{c}
i \\
j
\end{array}\right) \delta^{i-j} \varphi^{j}\left(\left\|S\left(\omega, u_{n+1}(\omega)\right)-T^{i}\left(\omega, u_{n+1}(\omega)\right)\right\|\right)\right) .
\end{aligned}
$$

From (20),

$$
\begin{gathered}
\left\|S\left(\omega, u_{n+1}(\omega)\right)-T^{i}\left(\omega, u_{n+1}(\omega)\right)\right\|=\| S\left(\omega, u_{n+1}(\omega)\right) \\
-S(\omega, p(\omega))+T(\omega, p(\omega))-T^{i}\left(\omega, u_{n+1}(\omega)\right) \| \\
\leq\left\|S\left(\omega, u_{n+1}(\omega)\right)-p(\omega)\right\|+\| T(\omega, p(\omega)) \\
-T^{i}\left(\omega, u_{n+1}(\omega)\right)\|\leq\| S\left(\omega, u_{n+1}(\omega)\right)-p(\omega) \|
\end{gathered}
$$

$$
\begin{aligned}
& +\delta^{i}\left\|p(\omega)-S\left(\omega, u_{n+1}(\omega)\right)\right\|+\left(\sum_{i=1}^{k_{1}} \alpha_{n, i}\right) \\
& \cdot\left(\sum_{j=0}^{i}\left(\begin{array}{l}
i \\
j
\end{array}\right) \delta^{i-j} \varphi^{j}(\|S(\omega, p(\omega))-T(\omega, p(\omega))\|)\right) \\
& =\left(1+\delta^{i}\right)\left\|S\left(\omega, u_{n+1}(\omega)\right)-p(\omega)\right\| .
\end{aligned}
$$


Substituting (21) into (20), we get

$$
\begin{aligned}
\left\|S\left(\omega, u_{n+1}(\omega)\right)-S\left(\omega, x_{n+1}(\omega)\right)\right\| \leq & \frac{\alpha_{n, 0}}{1-\sum_{i=1}^{k_{1}} \alpha_{n, i} \delta^{i}}\left\|S\left(\omega, u_{n}(\omega)\right)-S\left(\omega, x_{n}^{(1)}(\omega)\right)\right\| \\
& +\frac{\sum_{i=1}^{k_{1}} \alpha_{n, i} \sum_{j=0}^{i}\left(\begin{array}{c}
i \\
j
\end{array}\right) \delta^{i-j} \varphi^{j}\left(\left(1+\delta^{i}\right)\left\|S\left(\omega, u_{n+1}(\omega)\right)-p(\omega)\right\|\right)}{1-\sum_{i=1}^{k_{1}} \alpha_{n, i} \delta^{i}} .
\end{aligned}
$$

Using (11) and (14) and contractive condition (19), we get

$$
\begin{aligned}
& \left\|S\left(\omega, u_{n}(\omega)\right)-S\left(\omega, x_{n}^{(1)}(\omega)\right)\right\| \leq \beta_{n, 0}^{1} \| S\left(\omega, u_{n}(\omega)\right) \\
& -S\left(w, x_{n}^{(2)}(\omega)\right)\left\|+\sum_{i=1}^{q_{2}} \beta_{n, i}^{1}\right\| S\left(\omega, u_{n}(\omega)\right) \\
& -T^{i}\left(\omega, u_{n}(\omega)\right)+T^{i}\left(\omega, u_{n}(\omega)\right) \\
& -T^{i}\left(\omega, x_{n}^{(1)}(\omega)\right)\left\|\leq \beta_{n, 0}^{1}\right\| S\left(\omega, u_{n}(\omega)\right) \\
& -S\left(\omega, x_{n}^{(2)}(\omega)\right)\left\|+\sum_{i=1}^{q_{2}} \beta_{n, i}^{1}\right\| S\left(\omega, u_{n}(\omega)\right) \\
& -T^{i}\left(\omega, u_{n}(\omega)\right)\left\|+\sum_{i=1}^{k_{2}} \beta_{n, i}^{1}\right\| T^{i}\left(\omega, u_{n}(\omega)\right) \\
& -T^{i}\left(\omega, x_{n}^{1}(\omega)\right)\left\|\leq \beta_{n, 0}^{1}\right\| S\left(\omega, u_{n}(\omega)\right) \\
& -S\left(w, x_{n}^{(2)}(\omega)\right)\left\|+\sum_{i=1}^{q_{2}} \beta_{n, i}^{1}\right\| S\left(\omega, u_{n}(\omega)\right) \\
& -T^{i}\left(\omega, u_{n}(\omega)\right)\left\|+\left(\sum_{i=1}^{k_{2}} \beta_{n, i}^{1} \delta^{i}\right)\right\| S\left(\omega, u_{n}(\omega)\right) \\
& -S\left(\omega, x_{n}^{(1)}(\omega)\right) \|+\left(\sum_{i=1}^{k_{2}} \beta_{n, i}^{1}\right) \sum_{j=0}^{i}\left(\begin{array}{l}
i \\
j
\end{array}\right) \delta^{i-j} \varphi^{j} \\
& \cdot\left(\left\|S\left(\omega, u_{n}(\omega)\right)-T^{i}\left(\omega, u_{n}(\omega)\right)\right\|\right) .
\end{aligned}
$$

Following the method of proof in (21), we can write

$$
\begin{aligned}
& \left\|S\left(\omega, u_{n}(\omega)\right)-T^{i}\left(\omega, u_{n}(\omega)\right)\right\| \\
& \quad \leq\left(1+\delta^{i}\right)\left\|S\left(\omega, u_{n}(\omega)\right)-p(\omega)\right\| .
\end{aligned}
$$

Substituting (24) into (23) and simplifying, we obtain

$$
\begin{aligned}
& \left\|S\left(\omega, u_{n}(\omega)\right)-S\left(\omega, x_{n}^{(1)}(\omega)\right)\right\| \\
& \quad \leq \frac{\beta_{n, 0}^{1}}{1-\sum_{i=1}^{k_{2}} \beta_{n, i}^{1} \delta^{i}}\left\|S\left(\omega, u_{n}(\omega)\right)-S\left(\omega, x_{n}^{(2)}(\omega)\right)\right\| \\
& \quad+\frac{\sum_{i=1}^{k_{2}} \beta_{n, i}^{1}\left(1+\delta^{i}\right)}{1-\sum_{i=1}^{q_{2}} \beta_{n, i}^{1} \delta^{i}}\left\|S\left(\omega, u_{n}(\omega)\right)-p(\omega)\right\| \\
& +\frac{\sum_{i=1}^{k_{2}} \beta_{n, i}^{1} \sum_{j=0}^{i}\left(\begin{array}{c}
i \\
j
\end{array}\right) \delta^{i-j} \varphi^{j}\left(\left(1+\delta^{i}\right)\left\|S\left(\omega, u_{n}(\omega)\right)-p(\omega)\right\|\right)}{1-\sum_{i=1}^{k_{2}} \beta_{n, i}^{1} \delta^{i}} .
\end{aligned}
$$

Also, using (11) and (14) and contractive condition (19), we get

$$
\begin{aligned}
& \left\|S\left(\omega, u_{n}(\omega)\right)-S\left(\omega, x_{n}^{(2)}(\omega)\right)\right\| \\
& \quad \leq \frac{\beta_{n, 0}^{2}}{1-\sum_{i=1}^{k_{3}} \beta_{n, i}^{2} \delta^{i}}\left\|S\left(\omega, u_{n}(\omega)\right)-S\left(\omega, x_{n}^{(3)}(\omega)\right)\right\| \\
& +\frac{\sum_{i=1}^{k_{3}} \beta_{n, i}^{2}\left(1+\delta^{i}\right)}{1-\sum_{i=1}^{q_{3}} \beta_{n, i}^{2} \delta^{i}}\left\|S\left(\omega, u_{n}(\omega)\right)-p(\omega)\right\| \\
& +\frac{\sum_{i=1}^{k_{3}} \beta_{n, i}^{2} \sum_{j=0}^{i}\left(\begin{array}{c}
i \\
j
\end{array}\right) \delta^{i-j} \varphi^{j}\left(\left(1+\delta^{i}\right)\left\|S\left(\omega, u_{n}(\omega)\right)-p(\omega)\right\|\right)}{1-\sum_{i=1}^{k_{3}} \beta_{n, i}^{2} \delta^{i}} .
\end{aligned}
$$

Substituting (25) and (26) into (22), we get

$$
\begin{aligned}
& \left\|S\left(\omega, u_{n+1}(\omega)\right)-S\left(\omega, x_{n+1}(\omega)\right)\right\| \\
& \leq\left(\frac{\alpha_{n, 0}}{1-\sum_{i=1}^{k_{1}} \alpha_{n, i} \delta^{i}}\right)\left(\frac{\beta_{n, 0}^{1}}{1-\sum_{i=1}^{k_{2}} \beta_{n, i}^{1} \delta^{i}}\right)\left(\frac{\beta_{n, 0}^{2}}{1-\sum_{i=1}^{k_{3}} \beta_{n, i}^{2} \delta^{i}}\right)\left\|S\left(\omega, u_{n}(\omega)\right)-S\left(\omega, x_{n}^{(3)}(\omega)\right)\right\| \\
& \quad+\left(\frac{\alpha_{n, 0}}{1-\sum_{i=1}^{k_{1}} \alpha_{n, i} \delta^{i}}\right)\left(\frac{\beta_{n, 0}^{1}}{1-\sum_{i=1}^{k_{2}} \beta_{n, i}^{1} \delta^{i}}\right) \frac{\sum_{i=1}^{k_{3}} \beta_{n, i}^{2}\left(1+\delta^{i}\right)}{1-\sum_{i=1}^{k_{3}} \beta_{n, i}^{2} \delta^{i}}\left\|S\left(\omega, u_{n}(\omega)\right)-p(\omega)\right\| \\
& \quad+\left(\frac{\alpha_{n, 0}}{1-\sum_{i=1}^{k_{1}} \alpha_{n, i} \delta^{i}}\right)\left(\frac{\beta_{n, 0}^{1}}{1-\sum_{i=1}^{k_{2}} \beta_{n, i}^{1} \delta^{i}}\right)\left(\frac{\sum_{i=1}^{k_{3}} \beta_{n, i}^{2} \sum_{j=0}^{i}\left(\begin{array}{l}
i \\
j
\end{array}\right) \delta^{i-j} \varphi^{j}\left(\left(1+\delta^{i}\right)\left\|S\left(\omega, u_{n}(\omega)\right)-p(\omega)\right\|\right)}{1-\sum_{i=1}^{k_{3}} \beta_{n, i}^{2} \delta^{i}}\right)
\end{aligned}
$$


International Journal of Analysis

7

$$
\begin{aligned}
& +\left(\frac{\alpha_{n, 0}}{1-\sum_{i=1}^{k_{1}} \alpha_{n, i} \delta^{i}}\right)\left(\frac{\sum_{i=1}^{k_{2}} \beta_{n, i}^{1}\left(1+\delta^{i}\right)}{1-\sum_{i=1}^{k_{2}} \beta_{n, i}^{1} \delta^{i}}\right)\left\|S\left(\omega, u_{n}(\omega)\right)-p(\omega)\right\| \\
& +\left(\frac{\alpha_{n, 0}}{1-\sum_{i=1}^{k_{1}} \alpha_{n, i} \delta^{i}}\right)\left(\frac{\sum_{i=1}^{q_{2}} \beta_{n, i}^{1} \sum_{j=0}^{i}\left(\begin{array}{c}
i \\
j
\end{array}\right) \delta^{i-j} \varphi^{j}\left(\left(1+\delta^{i}\right)\left\|S\left(\omega, u_{n}(\omega)\right)-p(\omega)\right\|\right)}{1-\sum_{i=1}^{k_{2}} \beta_{n, i}^{(1)} \delta^{i}}\right) \\
& +\frac{\sum_{i=1}^{k_{1}} \alpha_{n, i} \sum_{j=0}^{i}\left(\begin{array}{c}
i \\
j
\end{array}\right) \delta^{i-j} \varphi^{j}\left(\left(1+\delta^{i}\right)\left\|S\left(\omega, u_{n+1}(\omega)\right)-p(\omega)\right\|\right)}{1-\sum_{i=1}^{k_{1}} \alpha_{n, i} \delta^{i}} .
\end{aligned}
$$

Continuing this process to $(q-1)$ and simplifying, we get the following:

$$
\begin{aligned}
& \left\|S\left(\omega, u_{n+1}(\omega)\right)-S\left(\omega, x_{n+1}(\omega)\right)\right\| \leq\left(\frac{\alpha_{n, 0}}{1-\sum_{i=1}^{k_{1}} \alpha_{n, i} \delta^{i}}\right)\left(\frac{\beta_{n, 0}^{1}}{1-\sum_{i=1}^{k_{2}} \beta_{n, i}^{1} \delta^{i}}\right)\left(\frac{\beta_{n, 0}^{2}}{1-\sum_{i=1}^{k_{3}} \beta_{n, i}^{2} \delta^{i}}\right) \cdots\left(\frac{\beta_{n, 0}^{q-2}}{1-\sum_{i=1}^{k_{q-1}} \beta_{n, i}^{q-2} \delta^{i}}\right) \\
& \cdot\left(\frac{\beta_{n, 0}^{q-1}}{1-\sum_{i=1}^{k_{q}} \beta_{n, i}^{q-1} \delta^{i}}\right)\left\|S\left(\omega, u_{n}(\omega)\right)-S\left(\omega, x_{n}(\omega)\right)\right\| \\
& +\left[\left(\frac{\beta_{n, 0}^{1}}{1-\sum_{i=1}^{k_{2}} \beta_{n, i}^{(1)} \delta^{i}}\right)\left(\frac{\beta_{n, 0}^{2}}{1-\sum_{i=1}^{k_{3}} \beta_{n, i}^{2} \delta^{i}}\right) \cdots\left(\frac{\beta_{n, 0}^{q-2}}{1-\sum_{i=1}^{k_{q-1}} \beta_{n, i}^{q-2} \delta^{i}}\right)\left(\frac{\sum_{i=1}^{k_{q}} \beta_{n, i}^{q-1}\left(1+\delta^{i}\right)}{1-\sum_{i=1}^{k_{q}} \beta_{n, i}^{q-1} \delta^{i}}\right)\right. \\
& \left.+\left(\frac{\beta_{n, 0}^{(1)}}{1-\sum_{i=1}^{k_{2}} \beta_{n, i}^{1} \delta^{i}}\right) \cdots\left(\frac{\beta_{n, 0}^{q-2}}{1-\sum_{i=1}^{k_{q-1}} \beta_{n, i}^{q-2} \delta^{i}}\right)\left(\frac{\sum_{i=1}^{k_{q-1}} \beta_{n, i}^{q-2}\left(1+\delta^{i}\right)}{1-\sum_{i=1}^{k_{q-1}} \beta_{n, i}^{q-2} \delta^{i}}\right)+\left(\frac{\sum_{i=1}^{k_{2}} \beta_{n, i}^{1}\left(1+\delta^{i}\right)}{1-\sum_{i=1}^{k_{2}} \beta_{n, i}^{1} \delta^{i}}\right)\right]\left(\frac{\alpha_{n, 0}}{1-\sum_{i=1}^{k_{1}} \alpha_{n, i} \delta^{i}}\right) \\
& \cdot\left\|S\left(\omega, u_{n}(\omega)\right)-p(\omega)\right\|+\left(\frac{\alpha_{n, 0}}{1-\sum_{i=1}^{k_{1}} \alpha_{n, i} \delta^{i}}\right)\left(\frac{\beta_{n, 0}^{1}}{1-\sum_{i=1}^{k_{2}} \beta_{n, i}^{1} \delta^{i}}\right)\left(\frac{\beta_{n, 0}^{2}}{1-\sum_{i=1}^{k_{3}} \beta_{n, i}^{2} \delta^{i}}\right) \cdots\left(\frac{\beta_{n, 0}^{q-2}}{1-\sum_{i=1}^{k_{q-1}} \beta_{n, i}^{q-2} \delta^{i}}\right) \\
& \cdot\left(\frac{\sum_{i=1}^{k_{q}} \beta_{n, i}^{q-1} \sum_{j=0}^{i}\left(\begin{array}{c}
i \\
j
\end{array}\right) \delta^{i-j} \varphi^{j}\left(\left(1+\delta^{i}\right)\left\|S\left(\omega, u_{n}(\omega)\right)-p(\omega)\right\|\right)}{1-\sum_{i=1}^{k_{q}} \beta_{n, i}^{q-1} \delta^{i}}\right) \\
& +\left(\frac{\sum_{i=1}^{k_{1}} \alpha_{n, i} \sum_{j=0}^{i}\left(\begin{array}{l}
i \\
j
\end{array}\right) \delta^{i-j} \varphi^{j}\left(\left(1+\delta^{i}\right)\left\|S\left(\omega, u_{n+1}(\omega)\right)-p(\omega)\right\|\right)}{1-\sum_{i=1}^{k_{1}} \alpha_{n, i} \delta^{i}}\right)
\end{aligned}
$$

Recall that $\alpha_{n, 0} /\left(1-\sum_{i=1}^{q_{1}} \alpha_{n, i} \delta^{i}\right) \leq \sum_{i=1}^{q_{1}} \alpha_{n, i} \delta^{i}+\alpha_{n, 0}$.

Let $\delta^{i} \leq \delta<1$; then,

$$
\sum_{i=1}^{q_{1}} \alpha_{n, i} \delta^{i}+\alpha_{n, 0} \leq\left[\left(1-\alpha_{n, 0}\right) \delta+\alpha_{n, 0}\right] .
$$

Substituting (29) into (28), we get

$$
\begin{aligned}
& \left\|S\left(\omega, u_{n+1}(\omega)\right)-S\left(\omega, x_{n+1}(\omega)\right)\right\| \\
& \quad \leq\left[1-\lambda_{n}\right]\left\|S\left(\omega, u_{n}(\omega)\right)-S\left(\omega, x_{n}(\omega)\right)\right\|+e_{n},
\end{aligned}
$$

where $\lambda_{n}=\left(1-\alpha_{n, 0}\right)(1-\delta)$,

$$
\begin{aligned}
e_{n}= & \left\{\left[\left(1-\beta_{n, 0}^{1}\right) \delta+\beta_{n, 0}^{1}\right]\right. \\
& \cdot\left[\left(1-\beta_{n, 0}^{2}\right) \delta+\beta_{n, 0}^{2}\right] \cdots\left[\left(1-\beta_{n, 0}^{k-2}\right) \delta+\beta_{n, 0}^{q-2}\right] \\
& \cdot\left[\frac{\sum_{i=1}^{k_{q}} \beta_{n, i}^{q-1}}{1-\sum_{i=1}^{k_{q}} \beta_{n, i}^{q-1} \delta^{i}}\right]
\end{aligned}
$$




$$
\begin{aligned}
& +\left[\left(1-\beta_{n, 0}^{1}\right) \delta+\beta_{n, 0}^{1}\right] \cdots\left[\frac{\sum_{i=1}^{k_{q-1}} \beta_{n, i}^{q-2}}{1-\sum_{i=1}^{k_{q-1}} \beta_{n, i}^{k-2} \delta^{i}}\right] \\
& \left.+\left[\frac{\sum_{i=1}^{k_{2}} \beta_{n, i}^{1}}{1-\sum_{i=1}^{k_{2}} \beta_{n, i}^{1} \delta^{1}}\right]\right\}\left[\left(1-\alpha_{n, 0}\right) \delta+\alpha_{n, 0}\right]\left(1+\delta^{i}\right) \\
& \cdot\left\|S\left(\omega, u_{n}(\omega)\right)-p(\omega)\right\|+\left[\left(1-\alpha_{n, 0}\right) \delta\right. \\
& \left.+\alpha_{n, 0}\right]\left[\left(1-\beta_{n, 0}^{1}\right) \delta+\beta_{n, 0}^{1}\right] \cdots\left[\left(1-\beta_{n, 0}^{q-2}\right) \delta\right. \\
& \left.+\beta_{n, 0}^{q-2}\right]\left[\frac{\sum_{i=1}^{k_{q}} \beta_{n, i}^{k-1} \sum_{j=0}^{i}\left(\begin{array}{c}
i \\
j
\end{array}\right) \delta^{i-j}}{1-\sum_{i=1}^{k_{q}} \beta_{n, i}^{q-1} \delta^{i}}\right] \varphi^{j}\left(\left(1+\delta^{i}\right)\right. \\
& \left.\cdot\left\|S\left(\omega, u_{n}(\omega)\right) p(\omega)\right\|\right) .
\end{aligned}
$$

Applying Lemma 5 in (30), it follows that $\lim _{n \rightarrow \infty} \| S(\omega$, $\left.u_{n}(\omega)\right)-S\left(\omega, x_{n}(\omega)\right) \|=0$.

By assumption $\lim _{n \rightarrow \infty} S\left(\omega, u_{n}(\omega)\right)=p(\omega)$, then $\| S(\omega$, $\left.x_{n}(\omega)\right)-p(\omega)\|\leq\| S\left(\omega, u_{n}(\omega)\right)-S\left(\omega, x_{n}(\omega)\right)\|+\| S\left(\omega, u_{n}(\omega)\right)-$ $p(\omega) \| \rightarrow 0$ as $n \rightarrow \infty$, that is, $\lim _{n \rightarrow \infty} S\left(\omega, x_{n}(\omega)\right)=p(\omega)$.

Next, we show that (ii) $\rightarrow$ (i). Assume $\lim _{n \rightarrow \infty} S(\omega$, $\left.x_{n}(\omega)\right)=p(\omega)$; then, using (11) and (14) and contractive condition (19), we have

$$
\begin{aligned}
& \left\|S\left(\omega, x_{n+1}(\omega)\right)-S\left(\omega, u_{n+1}(\omega)\right)\right\| \\
& \quad \leq \alpha_{n, 0}\left\|S\left(\omega, x_{n}^{1}(\omega)\right)-S\left(\omega, u_{n}(\omega)\right)\right\|+\sum_{i=1}^{k_{1}} \alpha_{n, i} \\
& \quad \| T^{i}\left(\omega, x_{n+1}(\omega)\right)
\end{aligned}
$$

$$
\begin{aligned}
& -T^{i}\left(\omega, u_{n+1}(\omega)\right)\left\|\leq \alpha_{n, 0}\right\| S\left(\omega, x_{n}^{1}(\omega)\right) \\
& -S\left(\omega, u_{n}(\omega)\right)\left\|+\left(\sum_{i=1}^{k_{1}} \alpha_{n, i} \delta^{i}\right)\right\| S\left(\omega, x_{n+1}(\omega)\right) \\
& -S\left(\omega, u_{n+1}(\omega)\right) \|+\sum_{i=1}^{k_{1}} \alpha_{n, i}\left(\sum_{j=0}^{i}\left(\begin{array}{l}
i \\
j
\end{array}\right) \delta^{i-j} \varphi^{j}\right. \\
& \left.\cdot\left(\left\|S\left(\omega, x_{n+1}(\omega)\right)-T^{i}\left(\omega, x_{n+1}(\omega)\right)\right\|\right)\right) .
\end{aligned}
$$

From (32),

$$
\begin{aligned}
& \left\|S\left(\omega, x_{n+1}(\omega)\right)-T^{i}\left(\omega, x_{n+1}(\omega)\right)\right\|=\| S\left(\omega, x_{n+1}(\omega)\right) \\
& -S(\omega, p(\omega))+T(\omega, p(\omega))-T^{i}\left(\omega, x_{n+1}(\omega)\right) \| \\
& \leq\left\|S\left(\omega, x_{n+1}(\omega)\right)-p(\omega)\right\|+\| T(\omega, p(\omega)) \\
& -T^{i}\left(\omega, x_{n+1}(\omega)\right)\|\leq\| S\left(\omega, x_{n+1}(\omega)\right)-p(\omega) \| \\
& +\delta^{i}\left\|p(\omega)-S\left(\omega, x_{n+1}(\omega)\right)\right\|+\left(\sum_{i=1}^{k_{1}} \alpha_{n, i}\right) \\
& \cdot\left(\sum_{j=0}^{i}\left(\begin{array}{l}
i \\
j
\end{array}\right) \delta^{i-j} \varphi^{j}(\|S(\omega, p(\omega))-T(\omega, p(\omega))\|)\right) \\
& =\left(1+\delta^{i}\right)\left\|S\left(\omega, x_{n+1}(\omega)\right)-p(\omega)\right\| .
\end{aligned}
$$

Substituting (33) into (32) and simplifying, we obtain

$$
\begin{aligned}
\left\|S\left(\omega, x_{n+1}(\omega)\right)-S\left(\omega, u_{n+1}(\omega)\right)\right\| \leq & \frac{\alpha_{n, 0}}{1-\sum_{i=1}^{k_{1}} \alpha_{n, i} \delta^{i}}\left\|S\left(\omega, x_{n}^{1}(\omega)\right)-S\left(\omega, u_{n}(\omega)\right)\right\| \\
& +\frac{\sum_{i=1}^{k_{1}} \alpha_{n, i} \sum_{j=0}^{i}\left(\begin{array}{c}
i \\
j
\end{array}\right) \delta^{i-j} \varphi^{j}\left(\left(1+\delta^{i}\right)\left\|S\left(\omega, x_{n+1}(\omega)\right)-p(\omega)\right\|\right)}{1-\sum_{i=1}^{k_{1}} \alpha_{n, i} \delta^{i}} .
\end{aligned}
$$

Using (11) and (14) and contractive condition (19), we have

$$
\begin{array}{ll}
\left\|S\left(\omega, x_{n}^{1}(\omega)\right)-S\left(\omega, u_{n}(\omega)\right)\right\| & \leq\left(\frac{\beta_{n, 0}^{1}}{1-\sum_{i=1}^{k_{2}} \beta_{n, i}^{1}}\right)\left\|S\left(\omega, x_{n}^{2}(\omega)\right)-S\left(\omega, u_{n}(\omega)\right)\right\| \\
\leq \beta_{n, 0}^{1}\left\|S\left(\omega, x_{n}^{2}(\omega)\right)-S\left(\omega, u_{n}(\omega)\right)\right\|+\sum_{i=1}^{k_{2}} \beta_{n, i}^{1} & +\left(\frac{\sum_{i=1}^{k_{2}} \beta_{n, i}^{1}}{1-\sum_{i=1}^{k_{2}} \beta_{n, i}^{1}}\right) \\
\cdot\left\|T^{i}\left(\omega, x_{n}^{1}(\omega)\right)-S\left(\omega, x_{n}^{1}(\omega)\right)\right\|+\sum_{i=1}^{k_{2}} \beta_{n, i}^{1} & \cdot\left\|T^{i}\left(\omega, x_{n}^{1}(\omega)\right)-S\left(\omega, x_{n}^{1}(\omega)\right)\right\| . \\
\cdot\left\|S\left(\omega, x_{n}^{1}(\omega)\right)-S\left(\omega, u_{n}(\omega)\right)\right\| &
\end{array}
$$


International Journal of Analysis

9

From (35),

$$
\begin{aligned}
& \left\|S\left(\omega, x_{n}^{1}(\omega)\right)-T^{i}\left(\omega, x_{n}^{1}(\omega)\right)\right\|=\| S\left(\omega, x_{n}^{1}(\omega)\right) \\
& -S(\omega, p(\omega))+T(\omega, p(\omega))-T^{i}\left(\omega, x_{n}^{1}(\omega)\right) \| \\
& \leq\left\|S\left(\omega, x_{n}^{1}(\omega)\right)-p(\omega)\right\|+\| T(\omega, p(\omega)) \\
& -T^{i}\left(\omega, x_{n}^{1}(\omega)\right)\|\leq\| S\left(\omega, x_{n}^{1}(\omega)\right)-p(\omega) \| \\
& +\delta^{i}\left\|S\left(\omega, x_{n}^{1}(\omega)\right)-p(\omega)\right\|+\sum_{j=0}^{i}\left(\begin{array}{l}
i \\
j
\end{array}\right) \delta^{i-j} \\
& \cdot \varphi^{j}(\|S(\omega, p(\omega))-T(\omega, p(\omega))\|)=\left(1+\delta^{i}\right) \\
& \text {. }\left\|S\left(\omega, x_{n}^{1}(\omega)\right)-p(\omega)\right\|, \\
& \left\|S\left(\omega, x_{n}^{1}(\omega)\right)-p(\omega)\right\| \leq \beta_{n, 0}^{1}\left\|S\left(\omega, x_{n}^{2}(\omega)\right)-p(\omega)\right\| \\
& +\sum_{i=1}^{k_{2}} \beta_{n, i}^{1}\left\|T^{i}\left(\omega, x_{n}^{1}(\omega)\right)-p(\omega)\right\| \\
& \leq\left(\frac{\beta_{n, 0}^{1}}{1-\sum_{i=1}^{k_{2}} \beta_{n, i}^{1} \delta^{i}}\right)\left\|S\left(\omega, x_{n}^{2}(\omega)\right)-p(\omega)\right\|, \\
& \left\|S\left(\omega, x_{n}^{2}(\omega)\right)-p(\omega)\right\| \leq\left(\frac{\beta_{n, 0}^{2}}{1-\sum_{i=1}^{k_{3}} \beta_{n, i}^{2} \delta^{i}}\right) \\
& \cdot\left(\frac{\beta_{n, 0}^{3}}{1-\sum_{i=1}^{k_{4}} \beta_{n, i}^{3} \delta^{i}}\right) \cdots\left(\frac{\beta_{n, 0}^{q-2}}{1-\sum_{i=1}^{k_{q-1}} \beta_{n, i}^{q-2} \delta^{i}}\right) \\
& \cdot\left(\frac{\beta_{n, 0}^{q-1}}{1-\sum_{i=1}^{k_{q}} \beta_{n, i}^{q-1} \delta^{i}}\right)\left\|S\left(\omega, x_{n}(\omega)\right)-p(\omega)\right\|, \\
& \left\|S\left(\omega, x_{n}^{q-1}(\omega)\right)-p(\omega)\right\| \leq\left(\frac{\beta_{n, 0}^{q-1}}{1-\sum_{i=1}^{k_{q}} \beta_{n, i}^{q-1} \delta^{i}}\right) \\
& \cdot\left\|S\left(\omega, x_{n}(\omega)\right)-p(\omega)\right\| .
\end{aligned}
$$

(36)

Substituting (37) and (38) into (36) yields

$$
\begin{aligned}
& \left\|S\left(\omega, x_{n}^{1}(\omega)\right)-T^{i}\left(\omega, x_{n}^{1}(\omega)\right)\right\| \leq\left(1+\delta^{i}\right) \\
& \quad \cdot\left(\frac{\beta_{n, 0}^{1}}{1-\sum_{i=1}^{k_{2}} \beta_{n, i}^{1} \delta^{i}}\right) \\
& \quad \cdot\left(\frac{\beta_{n, 0}^{2}}{1-\sum_{i=1}^{k_{3}} \beta_{n, i}^{2} \delta^{i}}\right) \cdots\left(\frac{\beta_{n, 0}^{q-2}}{1-\sum_{i=1}^{k_{q-1}} \beta_{n, i}^{q-2} \delta^{i}}\right) \\
& \quad\left(\frac{\beta_{n, 0}^{q-1}}{1-\sum_{i=1}^{k_{q}} \beta_{n, i}^{q-1} \delta^{i}}\right)\left\|S\left(\omega, x_{n}(\omega)\right)-p(\omega)\right\| .
\end{aligned}
$$

Substituting (40) into (35), we obtain

$$
\begin{aligned}
& \left\|S\left(\omega, x_{n}^{1}(\omega)\right)-S\left(\omega, u_{n}(\omega)\right)\right\| \leq\left(\frac{\beta_{n, 0}^{1}}{1-\sum_{i=1}^{k_{2}} \beta_{n, i}^{1}}\right) \\
& \cdot\left\|S\left(\omega, x_{n}^{2}(\omega)\right)-S\left(\omega, u_{n}(\omega)\right)\right\|+\left(1+\delta^{i}\right) \\
& \cdot\left(\frac{\beta_{n, 0}^{1}}{1-\sum_{i=1}^{k_{2}} \beta_{n, i}^{1} \delta^{i}}\right) \\
& \cdot\left(\frac{\beta_{n, 0}^{2}}{1-\sum_{i=1}^{k_{3}} \beta_{n, i}^{2} \delta^{i}}\right) \cdots\left(\frac{\beta_{n, 0}^{q-2}}{1-\sum_{i=1}^{k_{q-1}} \beta_{n, i}^{q-2} \delta^{i}}\right) \\
& \cdot\left(\frac{\beta_{n, 0}^{q-1}}{1-\sum_{i=1}^{k_{q}} \beta_{n, i}^{q-1} \delta^{i}}\right)\left(\frac{\sum_{i=1}^{k_{2}} \beta_{n, i}^{1}}{1-\sum_{i=1}^{k_{2}} \beta_{n, i}^{1}}\right) \\
& \cdot\left\|S\left(\omega, x_{n}(\omega)\right)-p(\omega)\right\| .
\end{aligned}
$$

$\left\|S\left(\omega, x_{n}^{2}(\omega)\right)-S\left(\omega, u_{n}(\omega)\right)\right\|, \ldots,\left\|S\left(\omega, x_{n}^{q-2}(\omega)\right)-S\left(\omega, u_{n}(\omega)\right)\right\|$, and $\left\|S\left(\omega, x_{n}^{q-1}(\omega)\right)-S\left(\omega, u_{n}(\omega)\right)\right\|$ are similarly obtained and substituted into (41) to get

$$
\begin{aligned}
& \left\|S\left(\omega, x_{n}^{1}(\omega)\right)-S\left(\omega, u_{n}(\omega)\right)\right\| \leq\left(\frac{\beta_{n, 0}^{1}}{1-\sum_{i=1}^{k_{2}} \beta_{n, i}^{1} \delta^{i}}\right) \\
& \cdot\left(\frac{\beta_{n, 0}^{2}}{1-\sum_{i=1}^{k_{3}} \beta_{n, i}^{2} \delta^{i}}\right) \cdots\left(\frac{\beta_{n, 0}^{q-2}}{1-\sum_{i=1}^{k_{q-1}} \beta_{n, i}^{q-2} \delta^{i}}\right) \\
& \cdot\left(\frac{\sum_{i=1}^{k_{q}} \beta_{n, i}^{k-1}}{1-\sum_{i=1}^{k_{q}} \beta_{n, i}^{q-1} \delta^{i}}\right) \| S\left(\omega, x_{n}(\omega)\right) \\
& -S\left(\omega, u_{n}(\omega)\right) \|+\left[\left(\frac{\sum_{i=1}^{k_{2}} \beta_{n, i}^{1}}{1-\sum_{i=1}^{k_{2}} \beta_{n, i}^{1}}\right)\right. \\
& \left.+\left(\frac{\sum_{i=1}^{k_{3}} \beta_{n, i}^{2}}{1-\sum_{i=1}^{k_{3}} \beta_{n, i}^{2} \delta^{i}}\right)+\cdots+\left(\frac{\sum_{i=1}^{k_{q}} \beta_{n, i}^{k-1}}{1-\sum_{i=1}^{k_{q}} \beta_{n, i}^{q-1} \delta^{i}}\right)\right] \\
& \cdot\left(\frac{\beta_{n, 0}^{1}}{1-\sum_{i=1}^{k_{2}} \beta_{n, i}^{1} \delta^{i}}\right)\left(\frac{\beta_{n, 0}^{2}}{1-\sum_{i=1}^{k_{3}} \beta_{n, i}^{2} \delta^{i}}\right) \\
& \ldots\left(\frac{\beta_{n, 0}^{q-2}}{1-\sum_{i=1}^{k_{q-1}} \beta_{n, i}^{q-2} \delta^{i}}\right)\left(\frac{\beta_{n, 0}^{q-1}}{1-\sum_{i=1}^{k_{q}} \beta_{n, i}^{q-1} \delta^{i}}\right)(1 \\
& \left.+\delta^{i}\right)\left\|S\left(\omega, x_{n}(\omega)\right)-p(\omega)\right\| .
\end{aligned}
$$


Substituting (42) into (34), we have

$$
\begin{aligned}
& \left\|S\left(\omega, x_{n+1}(\omega)\right)-S\left(\omega, u_{n+1}(\omega)\right)\right\| \leq\left(\frac{\alpha_{n, 0}}{1-\sum_{i=1}^{k_{1}} \alpha_{n, i} \delta^{i}}\right)\left(\frac{\beta_{n, 0}^{1}}{1-\sum_{i=1}^{k_{2}} \beta_{n, i}^{1} \delta^{i}}\right)\left(\frac{\beta_{n, 0}^{2}}{1-\sum_{i=1}^{k_{3}} \beta_{n, i}^{2} \delta^{i}}\right) \cdots\left(\frac{\beta_{n, 0}^{q-2}}{1-\sum_{i=1}^{k_{q-1}} \beta_{n, i}^{q-2} \delta^{i}}\right) \\
& \cdot\left(\frac{\sum_{i=1}^{k_{q}} \beta_{n, i}^{q-1}}{1-\sum_{i=1}^{k_{q}} \beta_{n, i}^{q-1} \delta^{i}}\right)\left\|S\left(\omega, x_{n}(\omega)\right)-S\left(\omega, u_{n}(\omega)\right)\right\| \\
& +\left[\left(\frac{\sum_{i=1}^{k_{2}} \beta_{n, i}^{1}}{1-\sum_{i=1}^{k_{2}} \beta_{n, i}^{1}}\right)+\left(\frac{\sum_{i=1}^{k_{3}} \beta_{n, i}^{2}}{1-\sum_{i=1}^{k_{3}} \beta_{n, i}^{2} \delta^{i}}\right)+\cdots+\left(\frac{\sum_{i=1}^{k_{q}} \beta_{n, i}^{q-1}}{1-\sum_{i=1}^{k_{q}} \beta_{n, i}^{q-1} \delta^{i}}\right)\right] \\
& \cdot\left(\frac{\alpha_{n, 0}}{1-\sum_{i=1}^{k_{1}} \alpha_{n, i} \delta^{i}}\right)\left(\frac{\beta_{n, 0}^{1}}{1-\sum_{i=1}^{k_{2}} \beta_{n, i}^{1} \delta^{i}}\right)\left(\frac{\beta_{n, 0}^{2}}{1-\sum_{i=1}^{k_{3}} \beta_{n, i}^{2} \delta^{i}}\right) \\
& \cdots\left(\frac{\beta_{n, 0}^{q-2}}{1-\sum_{i=1}^{k_{q-1}} \beta_{n, i}^{q-2} \delta^{i}}\right)\left(\frac{\beta_{n, 0}^{q-1}}{1-\sum_{i=1}^{k_{q}} \beta_{n, i}^{q-1} \delta^{i}}\right)\left(1+\delta^{i}\right)\left\|S\left(\omega, x_{n}(\omega)\right)-p(\omega)\right\| \\
& +\frac{\sum_{i=1}^{k_{1}} \alpha_{n, i} \delta^{i} \sum_{j=0}^{i}\left(\begin{array}{c}
i \\
j
\end{array}\right) \delta^{i-j} \varphi^{j}\left(\left(1+\delta^{i}\right)\left\|S\left(\omega, x_{n+1}(\omega)\right)-p(\omega)\right\|\right)}{1-\sum_{i=1}^{k_{1}} \alpha_{n, i} \delta^{i}} .
\end{aligned}
$$

Recall that $\alpha_{n, 0} /\left(1-\sum_{i=1}^{k_{1}} \alpha_{n, i} \delta^{i}\right) \leq \sum_{i=1}^{k_{1}} \alpha_{n, i} \delta^{i}+\alpha_{n, 0}$.

Let $\delta^{i} \leq \delta<1$; then,

$$
\sum_{i=1}^{k_{1}} \alpha_{n, i} \delta^{i}+\alpha_{n, 0} \leq\left[\left(1-\alpha_{n, 0}\right) \delta+\alpha_{n, 0}\right] .
$$

Using (44) in (43), we have

$$
\begin{aligned}
& \left\|S\left(\omega, x_{n+1}(\omega)\right)-S\left(\omega, u_{n+1}(\omega)\right)\right\| \\
& \quad \leq\left[1-\lambda_{n}\right]\left\|S\left(\omega, x_{n}(\omega)\right)-S\left(\omega, u_{n}(\omega)\right)\right\|+e_{n},
\end{aligned}
$$

where $\lambda_{n}=\left(1-\alpha_{n, 0}\right)(1-\delta)$,

$$
\begin{aligned}
e_{n}= & \left(1+\delta^{i}\right)\left[\left(1-\alpha_{n, 0}\right) \delta+\alpha_{n, 0}\right]\left[\left(1-\beta_{n, 0}^{1}\right) \delta+\beta_{n, 0}^{1}\right]\left[\left(1-\beta_{n, 0}^{2}\right) \delta+\beta_{n, 0}^{2}\right] \cdots\left[\left(1-\beta_{n, 0}^{q-2}\right) \delta+\beta_{n, 0}^{q-2}\right] \\
& \cdot\left[\left(\frac{\sum_{i=1}^{q_{2}} \beta_{n, i}^{1}}{1-\sum_{i=1}^{k_{2}} \beta_{n, i}^{1}}\right)+\left(\frac{\sum_{i=1}^{k_{3}} \beta_{n, i}^{2}}{1-\sum_{i=1}^{k_{3}} \beta_{n, i}^{2} \delta^{i}}\right)+\cdots+\left(\frac{\sum_{i=1}^{k_{q}} \beta_{n, i}^{q-1}}{1-\sum_{i=1}^{k_{q}} \beta_{n, i}^{q-1} \delta^{i}}\right)\right]\left\|S\left(\omega, x_{n}(\omega)\right)-p(\omega)\right\| \\
& +\frac{\sum_{i=1}^{k_{1}} \alpha_{n, i} \delta^{i} \sum_{j=0}^{i}\left(\begin{array}{c}
i \\
j
\end{array}\right) \delta^{i-j} \varphi^{j}\left(\left(1+\delta^{i}\right)\left\|S\left(\omega, x_{n+1}(\omega)\right)-p(\omega)\right\|\right)}{1-\sum_{i=1}^{k_{1}} \alpha_{n, i} \delta^{i}} .
\end{aligned}
$$

Using Lemma 5 in (45), it follows that $\lim _{n \rightarrow \infty} \| S\left(\omega, x_{n}(\omega)\right)-$ $S\left(\omega, u_{n}(\omega)\right) \|=0$.

Since, by assumption, $\lim _{n \rightarrow \infty} S\left(\omega, x_{n}(\omega)\right)=p(\omega)$, then $\left\|S\left(\omega, u_{n}(\omega)\right)-p(\omega)\right\| \leq\left\|S\left(\omega, x_{n}(\omega)\right)-S\left(\omega, u_{n}(\omega)\right)\right\|+\| S(\omega$, $\left.x_{n}(\omega)\right)-p(\omega) \| \rightarrow 0$ as $n \rightarrow \infty$; that is, $\lim _{n \rightarrow \infty} S\left(\omega, u_{n}(\omega)\right)=$ $p(\omega)$.

Since (i) $\rightarrow$ (ii) and (ii) $\rightarrow$ (i), it is shown that the convergence of random implicit Jungck-Kirk-Mann iteration (14) is equivalent to the convergence of random implicit Jungck-Kirk-multistep iteration (11) when applied to a pair of random weakly compatible generalized $\varphi$-contractive-like conditions (19). This ends the proof.

Since the random implicit Jungck-Kirk-multistep iteration (11) generalizes other random implicit Jungck-Kirk-type iterations (12), (13), and (14), then Theorem 7 leads to the following corollaries.

Corollary 8. Let $E$ be a separable Banach space and $S, T$ : $\Omega \times E \rightarrow E$ be two random commuting mappings satisfying (19) such that $T(\omega, E) \subseteq S(\omega, E)$. Assume that $S$ and $T$ are random weakly compatible. Let $p(\omega)$ be the random common 
TABLE 1: Approximation of stochastic common fixed point.

\begin{tabular}{ccccccccc}
\hline$n$ & $x_{n}$ & $y_{n}$ & $z_{n}$ & $u_{n}$ & $S\left(\omega, x_{n}(\omega)\right)$ & $S\left(\omega, y_{n}(\omega)\right)$ & $S\left(\omega, z_{n}(\omega)\right)$ & $S\left(\omega, u_{n}(\omega)\right)$ \\
\hline 0 & 0.1000 & 0.1000 & 0.1000 & 0.1000 & 0.1000 & 0.1000 & 0.1000 \\
1 & 1.0472 & 1.0468 & 1.0462 & 1.0459 & 0.6982 & 0.6926 & 0.6912 & 0.1000 \\
2 & 1.0739 & 1.0711 & 1.0692 & 1.0673 & 0.7343 & 0.7337 & 0.7332 \\
$\vdots$ & $\vdots$ & $\vdots$ & $\vdots$ & $\vdots$ & $\vdots$ & $\vdots$ & $\vdots$ & 0.7328 \\
5 & 1.0790 & 1.0783 & 1.0740 & 1.0702 & 0.7411 & 0.7407 & $\vdots$ & 0.7404 \\
$\vdots$ & $\vdots$ & $\vdots$ & $\vdots$ & $\vdots$ & $\vdots$ & 0.7413 & 0.7412 & $\vdots$ \\
7 & 1.0791 & 1.0791 & 1.0769 & 1.0766 & 0.7414 & 0.7415 & 0.7414 \\
8 & 1.0792 & 1.0792 & 1.0791 & 1.0791 & 0.7415 & 0.7415 & 0.7411 \\
9 & 1.0792 & 1.0792 & 1.0792 & 1.0791 & 0.7415 & 0.7414 \\
\hline
\end{tabular}

point of $S, T, S^{i}, T^{i}$ (i.e., $\left.S(\omega, p(\omega))=T(\omega, p(\omega))=p(\omega)\right)$. If $u_{0}, z_{0}, y_{0} \in$ E defined by $\left\{S\left(\omega, u_{n}(\omega)\right)\right\}_{n=0}^{\infty},\left\{S\left(\omega, z_{n}(\omega)\right)\right\}_{n=0}^{\infty}$ and $\left\{S\left(\omega, y_{n}(\omega)\right)\right\}_{n=0}^{\infty}$ as sequences satisfying (14), (13), and (12), respectively, then the following are equivalent:

(a)

(i) Random implicit Jungck-Kirk-Mann iteration (14) converges strongly to $p(\omega)$.

(ii) Random implicit Jungck-Kirk-Ishikawa iteration (13) converges strongly to $p(\omega)$.

(b)

(i) Random implicit Jungck-Kirk-Mann iteration (14) converges strongly to $p$.

(ii) Random implicit Jungck-Kirk-Noor iteration (12) converges strongly to $p$.

Proof. The proof of Corollary 8 is similar to that of Theorem 7.

Corollary 9. Let $E$ be a separable Banach space and $S, T$ : $\Omega \times E \rightarrow E$ be two random commuting mappings satisfying (19) such that $T(\omega, E) \subseteq S(\omega, E)$. Assume that $S$ and $T$ are random weakly compatible. Let $p(\omega)$ be the random common point of $S, T, S^{i}, T^{i}$ (i.e., $\left.S(\omega, p(\omega))=T(\omega, p(\omega))=p(\omega)\right)$. If $u_{0}, z_{0}$, $y_{0}, x_{0} \in E$ defined by $\left\{S\left(\omega, u_{n}(\omega)\right)\right\}_{n=0}^{\infty},\left\{S\left(\omega, z_{n}(\omega)\right)\right\}_{n=0}^{\infty}$, $\left\{S\left(\omega, y_{n}(\omega)\right)\right\}_{n=0}^{\infty}$ and $\left\{S\left(\omega, x_{n}(\omega)\right)\right\}_{n=0}^{\infty}$ as sequences satisfying (14), (13), (12), and (11), respectively, then the following are equivalent:

(i) Random implicit Jungck-Kirk-Mann iteration (14) converges strongly to $p(\omega)$.

(ii) Random implicit Jungck-Kirk-Ishikawa iteration (13) converges strongly to $p(\omega)$.

(iii) Random implicit Jungck-Kirk-Noor iteration (12) converges strongly to $p(\omega)$.

(iv) Random implicit Jungck-Kirk-multistep iteration (11) converges strongly to $p(\omega)$.

Example 10. Consider the equation $f(\omega, x(\omega))=0$, where $f$ is a random real function defined on interval $[0, \pi / 2]$ by $f(\omega, x(\omega))=x^{2}(\omega)-(\pi / 2)^{2} \cos (x(\omega))$.
Note that $f$ can be decomposed as $f=(\pi / 2)(S-T)$, where the maps $S$ and $T$ are the self-mappings in $[0, \pi / 2]$ defined by $S\left((\omega, x(\omega)):=(2 / \pi) x^{2}(\omega)\right.$ and $T((\omega, x(\omega)):=$ $(\pi / 2) \cos (x(\omega))$. Clearly, $S((\omega, x(\omega))$ and $T((\omega, x(\omega))$ satisfy the generalized $\varphi$-contractive condition (19). They coincide at $a(\omega) \approx 1.0792$ and we have $p(\omega)=S(\omega, a(\omega))=T(\omega, a(\omega)) \approx$ 0.7415 . Thus, $a(\omega)$ is a solution to $f(\omega, x(\omega))=0$. However, if $S$ and $T$ are random weakly compatible, then $p(\omega)=$ $S(\omega, p(\omega))=T(\omega, p(\omega)) \approx 0.7415$.

Using MATLAB, we show in Table 1 that (11), (12), (13), and (14) are equivalently used to approximate the stochastic common fixed point $p(\omega)$.

\section{Conclusion}

In this work, we have investigated the equivalence of convergence of various stochastic implicit Jungck-Kirk-type iterations for generalized random contractive-like operators in separable Banach spaces. We also gave an example to show the equivalence results. We hope that the findings in this paper will help researchers enhance and promote the further study on iterative schemes for two or more maps in well known spaces to carry out a general framework for their applications in real life.

\section{Conflicts of Interest}

The authors declare that there are no conflicts of interest regarding the publication of this paper.

\section{Acknowledgments}

The authors are grateful to Professor J. O. Olaleru for supervising their Ph.D. thesis. The first author is also grateful to his second Ph.D. supervisor, Professor J. A. Adepoju.

\section{References}

[1] A. Spacek, "Zufallige Gleichungen,” Czechoslovak Mathematical Journal, vol. 5, no. 80, pp. 462-466, 1955 (German), with Russian summary. 
[2] O. Hans, "Reduzierende Zufallige transformationen," Czechoslovak Mathematical Journal, vol. 82, no. 7, pp. 154-158, 1957 (German), with Russian summary.

[3] O. Hans, "Random operator equations," in Proceedings of the 4th Berkeley Sympos. Math. Statist. and Prob., vol. II, pp. 185-202, University of California Press, CA, USA, 1961.

[4] R. A. Rashwan and H. A. Hammad, "Stability and strong convergence results for random Jungck-Kirk-Noor iterative scheme," Fasciculi Mathematici, no. 58, pp. 167-182, 2017.

[5] D. O'Regan, "A continuation type result for random operators," Proceedings of the American Mathematical Society, vol. 126, no. 7, pp. 1963-1971, 1998.

[6] M. C. Joshi and R. K. Bose, Some Topics in Nonlinear Functional Analysis, Wiley Eastern Limited, New Delhi, India, 1985.

[7] S. S. Zhang, Fixed Point Theory and Applications, Chongqing Publishing Press, Chongqing, China, 1984.

[8] H. Akewe, G. A. Okeke, and A. Olayiwola, "Strong convergence and stability of Kirk-multistep-type iterative schemes for contractive-type operators," Fixed Point Theory and Applications, vol. 45, 24 pages, 2014.

[9] H. Akewe and A. Mogbademu, "Common fixed point of JungckKirk-type iterations for non-self operators in normed linear spaces," Fasciculi Mathematici, no. 56, pp. 29-41, 2016.

[10] W. A. Kirk, "A fixed point theorem for mappings which do not increase distances," The American Mathematical Monthly, vol. 72, pp. 1004-1006, 1965.

[11] W. A. Kirk, "On successive approximations for nonexpansive mappings in Banach spaces," Glasgow Mathematical Journal, vol. 12, pp. 6-9, 1971.

[12] J. O. Olaleru and H. Akewe, "The convergence of Jungck-type iterative schemes for generalized contractive-like operators," Fasciculi Mathematici, no. 45, pp. 87-98, 2010.

[13] M. O. Olatinwo, "Some stability and strong convergence results for the Jungck-Ishikawa iteration process," Creative Mathematics and Informatics, vol. 17, pp. 33-42, 2008.

[14] V. O. Olisama, A. A. Mogbademu, and J. O. Olaleru, "Modified multi-step iterative processes for a finite family of asymptotically pseudocontrative maps," Applied Mathematical Sciences, vol. 19, no. 4, pp. 901-910, 2014.

[15] X. Weng, "Fixed point iteration for local strictly pseudocontractive mapping," Proceedings of the American Mathematical Society, vol. 113, no. 3, pp. 727-731, 1991.

[16] H. Akewe and G. A. Okeke, "Convergence and stability theorems for the Picard-Mann hybrid iterative scheme for a general class of contractive-like operators," Fixed Point Theory and Applications, vol. 2015, no. 66, 8 pages, 2015.

[17] H. Akewe and G. A. Okeke, "Stability results for multistep iteration satisfying a general contractive condition of integral type in a normed linear space," Journal of Nigerian Association of Mathematical Physics, vol. 20, pp. 5-12, 2012.

[18] I. Beg and M. Abbas, "Equivalence and stability of random fixed point iterative procedures," Journal of Applied Mathematics and Stochastic Analysis. JAMSA, Article ID 23297, pp. 1-19, 2006.

[19] A. Djoudi and A. Aliouche, "Common fixed point theorems of Gregus type for weakly compatible mappings satisfying contractive conditions of integral type," Journal of Mathematical Analysis and Applications, vol. 329, no. 1, pp. 31-45, 2007.

[20] J. O. Olaleru, "On the equivalence of Picard, Mann and Ishikawa iterations for a class of quasi-contractive operators," Journal of Nigerian Association of Mathematical Physics, vol. 11, no. 1, pp. 51-56, 2007.
[21] B. E. Rhoades and S. M. Soltuz, "The equivalence between Mann-Ishikawa iterations and multistep iteration," Nonlinear Analysis, vol. 58, pp. 219-228, 2004.

[22] S. M. Soltuz, "The equivalence of Picard, Mann and Ishikawa iterations dealing with quasi-contractive operators," Mathematical Communications, vol. 10, pp. 81-88, 2005.

[23] S. M. Soltuz, "The equivalence between Krasnoselskij, Mann, Ishikawa, Noor and multistep iterations," Mathematical Communications, vol. 12, pp. 53-61, 2007.

[24] Z. Xue, "Remarks of equivalence among Picard, Mann and Ishikawa iterations in normed spaces," Fixed Point Theory and Applications, Article ID 61434, pp. 1-5, 2007.

[25] J. O. Olaleru and H. Akewe, "The equivalence of Jungck-type iterations for generalized contractive-like operators in a Banach space," Fasciculi Mathematici, no. 47, pp. 47-61, 2011. 


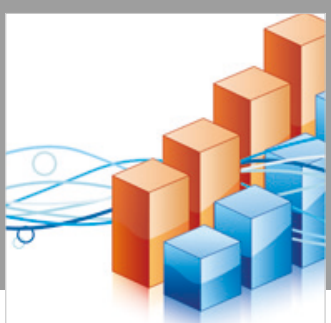

Advances in

Operations Research

\section{-n-m}
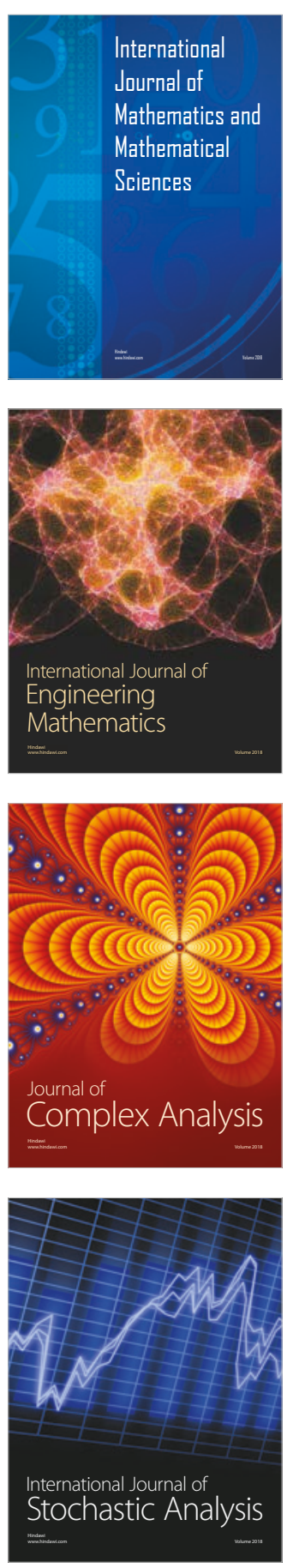
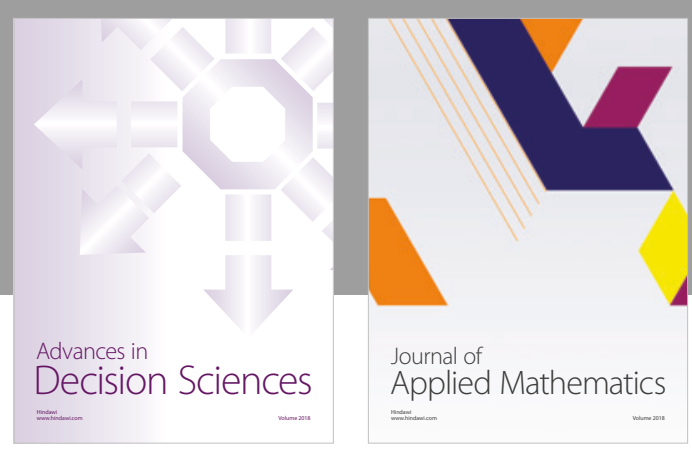

Journal of

Applied Mathematics
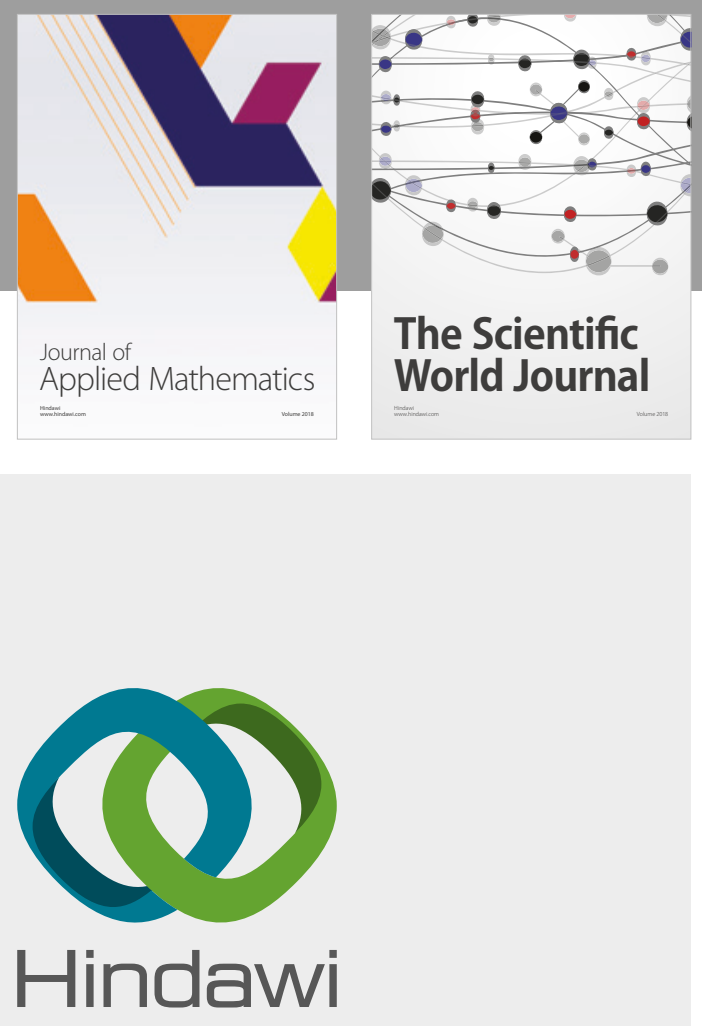

Submit your manuscripts at

www.hindawi.com

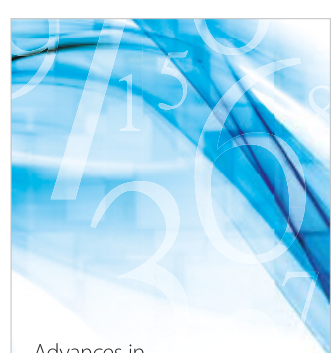

Advances in
Numerical Analysis
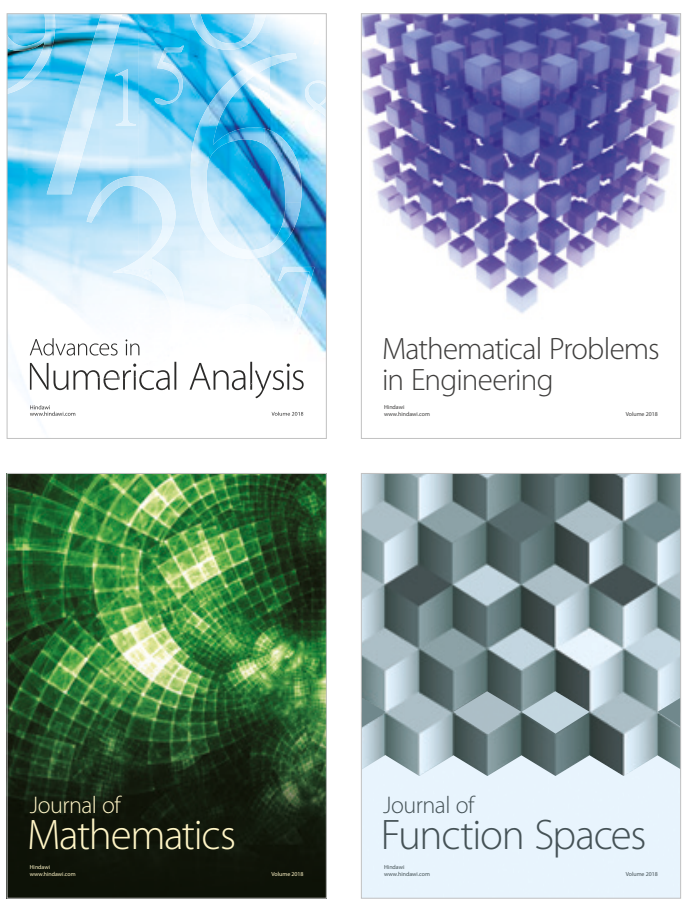

Mathematical Problems in Engineering

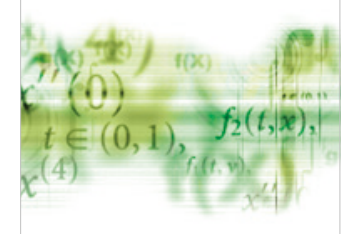

International Journal of

Differential Equations

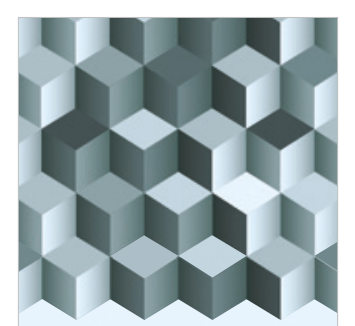

Journal of

Function Spaces

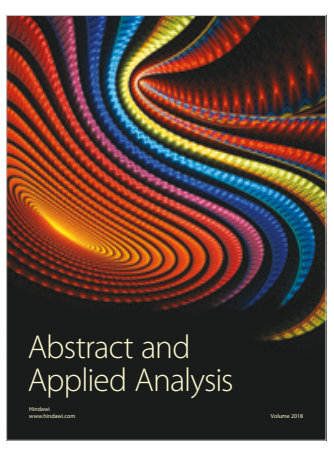

The Scientific

World Journal

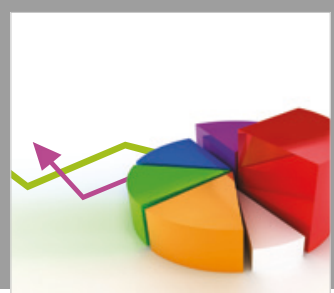

Journal of

Probability and Statistics
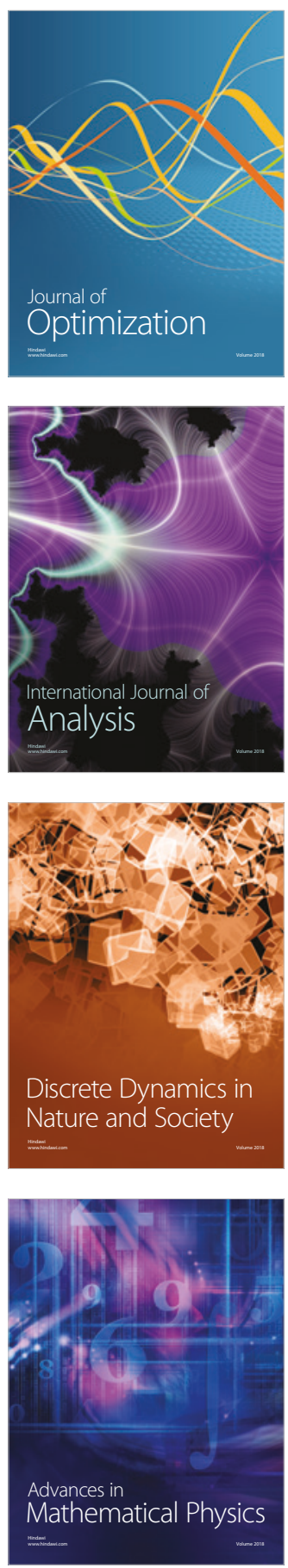